\title{
THE INTERACTION OF CLASSIFIERS AND SYNTAX IN SOUTH AFRICAN SIGN LANGUAGE
}

\author{
Debra Aarons, University of Stellenbosch, South Africa \\ Ruth Morgan, University of the Witwatersrand, South Africa
}

\section{Introduction}

In this paper ${ }^{1}$ we examine two aspects of constructions involving classifier handshapes in interaction with the syntax of an utterance. The first aspect we examine is the requirement that in South African Sign Language, classifier predicates ${ }^{2}$ are preceded by at least one element in the topic position ${ }^{3}$ of a sentence. Accordingly, we explore the syntactic constraints on sign order in the use of classifier predicates in South African Sign Language (henceforth SASL). Whereas it seems obvious that in signed language sentences involving classifier constructions, the lexical NPs should first. be identified, this requirement, and the constraints on this requirement have not really been explored in the literature. We know, for instance, that in American Sign Language (henceforth ASL) and SASL (at least) a sentence may not contain more than two topics (except in a listing construction) $)^{4}$. Many sentences containing classifier predicates have at least three lexical NPs thematically, e.g., in a signed language utterance with the English meaning, "The woman put the cup on the table", but only two of the NPs may occur as topics. We thus examine what may and may not appear in topic positions in these constructions (and whether or not these restrictions are determined by thematic roles). Further, we examine the syntactic relationship between classifier predicates and the items occurring in topic positions.

The second aspect we examine is the interaction of classifier predicates and what has been called role-play. In relation to this aspect, we describe and attempt to explain the constraints on the occurrence of role-play within utterances containing classifier predicates 5 .

We argue that what has often been described as role-play is a conflation of several different phenomena. One phenomenon is the use of the signer's hands to make what have been called handling classifiers (Supalla, 1982), or instrumental classifiers (Liddell and Johnson, 1987) as part of the complex predicate. We claim that this phenomenon involves the use of a linguistic item, a complex verb frame, in which the occurrence of the handshape may be seen as contributing semantic meaning and morphological agreement. The second phenomenon is the use of the head and shoulders of the signer to represent the head, and shoulders of the animate 
(person or animal) NP in the utterance. Supalla (1982) calls this the body classifier, Kegl (1985) calls it the signer's body pronoun. Engberg-Pedersen (1993) describes the use of role-shifted facial expressions as

"shifted attribution of expressive elements" and the shifted use of pronominal forms as "shifted reference". Many researchers argue against the label "classifier" for describing this phenomenon, or cluster of phenomena. (See Schembri, (in press)). Thirdly, there is a phenomenon in which miming actions and/or affective facial expressions accompany verbal items, either co-occurring with them, or following them.

We argue that the second and third phenomena, sometimes called role-play, or constructed action (Liddell and Metzger,1998) are not in themselves a necessary part of predicate classifier constructions, but provide, in fact, a technique for embellishing or extending description, once the signer has assumed the perspective of the NP in question. Following Liddell and Metzger (1998), we argue that role-play is not actually a part of the grammar, although we show that its occurrence, particularly in utterances involving classifier predicates, is subject to grammatical constraints. We show that when role-plays occur within utterances involving classifier predicates, they can never occur before the initial signing of the classifier predicate. They must either follow a classifier predicate, or they must occur simultaneously with a classifier predicate. Role-plays that follow a classifier predicate may also themselves be followed by a repetition of the initial classifier predicate.

\section{Topics and classifier predicates}

In SASL, as in ASL, the maximum number of allowable topic positions in a sentence is two. This excludes listing constructions. Topic positions in SASL, and in ASL, occur at the left edge of a sentence. Where topics appear to occur in other positions in a sentence, these are invariably embedded sentences, such as shown in the sentence below.

\section{top \\ 1. JOHN BELIEVE YESTERDAY VEGETABLE \\ INDEX BUY SPINACH \\ "John believes that yesterday, as far as vegetables are concerned, he bought spinach."}

Grammatical sentences in SASL may contain no topics, one topic or two topics. The order in which topics may occur depends on the sorts of topics they are semantically, their syntactic relationship to the rest of the sentence, and the non-manual marking that they bear, which is 
related to their semantic representation (Aarons 1994, 1996). We provide this information about topics and topic position in order to examine the relationship between NP topics and the use of classifier predicates.

When an NP is engaged in an action involving movement or location, it necessarily occurs at the left edge of a sentence, in either initial or second position, depending on how many NPs there are in the sentence. We have called these topic positions, although the NP may well be focused in terms of its function ${ }^{6}$. (Important to the discussion here is this particular position of the NP in the sentence, rather than its name). Such NPs are usually associated with a specific handshape depending on the perspective on that NP that the signer chooses. For simplicity, we call these classifier handshapes, although nothing hangs on the term. The complex verb frame of this sort of sentence (where the NP is engaged in an action involving movement or location) always includes the classifier handshape in addition to the movement, otherwise the sentence is not grammatical. Thus if the NP in question is a person, then the verb frame must involve a handshape that represents some aspect of a person. So, the handshape may be a $\mathrm{G}$ classifier (or an upright 1), or an $\mathrm{S}$ representing a person's head, or a handling classifier, showing that a person or a bodypart of a person is somehow involved in the action. First, we use simple examples, in which there is only one classifier handshape involved ${ }^{7}$.

\footnotetext{
top

2. MAN CL:G PERSON-MOVE- FORWARD

"The man walked."
}
3. MAN CL:S HEAD-MOVE SIDE-TO-SIDE-AS-PERSON-MOVES-FORWARD + "The man walked."
top
4. WOMAN
BABY CARRIAGE
CL:S(2 hands)
PERSON-PUSH-
CARRIAGE
"The woman pushed the baby carriage."

Example (4) demonstrates that the NPs under discussion are left-dislocated in some way and do not occur in the SVO order to be found in sentences containing plain verbs, i.e. verbs with no 
agreement marking, or no classifier handshape, such as (5). The incorrect sentence in (6) shows that such left-dislocation is not permissible, without topic marking over the left-dislocated element.

\section{MARY LOVE JOHN \\ "Mary loves John." \\ 6. "MARY JOHN LOVE (no topic marking) \\ "Mary loves John."}

So, as we have seen in (2)-(4), where there is a person NP in topic position, there must be a corresponding classifier handshape in the verb complex, and this classifier construction must in some way be associated with the person NP. This could well be accounted for as an agreement phenomenon. The same may be observed of animal NPs in topic position, where the verb complex must contain a classifier that is associated with that animal in topic position.

Generally, where there is a non-living object in topic position, the verb complex must contain a classifier associated with that object. This is shown in sentences (7)-(8) below, for one topic, and then for two.

top

7. AIRPLANE CL:Y FLY

"An airplane flies by."

\begin{tabular}{lll} 
8. & top & top \\
\cline { 2 - 2 } & CUP & LH CL:B (table) \\
& & RH CL:C (cup on table)
\end{tabular}

"The cup is on the table."

However, the picture is somewhat more complicated in the case where one of the NP topics is animate, and one is inanimate. Recall that the classifier handshape associated with the person NP must always occur in the verb complex. In sentences (9)-(10), we show examples where one NP topic is a person and the other an object. 

top top
9. BICYCLE-MOVE FORWARD 2H CL:S (alt.circles) PEDAL +++
"Meryl is riding a bicycle."
top
top
10. MERYL BICYCLE CL:X (palm down) BICYCLE-MOVE FORWARD 2H CL:S (alt.circles) PEDAL+++ CL:X (palm down) BICYCLE-MOVE FORWARD ++ "Meryl is riding a bicycle."

Note that when the order of the topics is reversed, as in (11) and (12), both the possible complex verb orders are available.

\footnotetext{
top top

11. BICYCLE MERYL 2H CL:S (alt.circles) PEDAL 2H CL:S (ait.circles) CL:X (palm down) BICYCLE-MOVE FORWARD 2H CL:S (alt.circles) PEDAL ++ "Meryl is riding a bicycle."
}
top top
12. BICYCLE MERYL CL:X (palm down) BICYCLE-MOVE FORWARD 2H CL:S (alt.circles) PEDAL ++ CL:X (palm down) BICYCLE-MOVE FORWARD ++ "Meryl is riding a bicycle."

Note, however, that sentences (13) and (14) are grammatical whereas sentences (15) and (16) are not.
13. $\frac{\text { top }}{\text { MERYL }}$
top
14. BICYCLE MERYL 2H CL:S (alt.circles) PEDAL +++ "Meryl is riding a bicycle."




\section{top top \\ 15. *MERYL BICYCLE CL:X (palm down) BICYCLE-MOVE-FORWARD++++
top top
16. "BICYCLE MERYL CL-X (palm down) BICYCLE-MOVE-FORWARD++++ "Meryl is riding a bicycle."

The important generalization that we can draw from these data is that when there is a person NP topic involved, whereas it is possible not to specifically encode the non-animate classifier in the verb complex, it is entirely ungrammatical not to encode the person classifier in the verb complex. In other words, the agreement with the person NP is crucial, and overrides (or incorporates) the grammatical necessity for the non-animate classifier to be encoded in the verb complex.

One might argue that the person involvement in the verb complex always incorporates the inanimate object semantically, so there is no need to overtly specify the inanimate object in cases like this ${ }^{8}$. Thus (even in English), cycling includes the notion that there is a bicycle. However, it is nonsensical in the sign language examples, to focus on Meryl, and then not involve her in the action. The only reading in which (17) would be grammatical is one in which Meryl is not on the bicycle.

\footnotetext{
17. $\frac{\text { top }}{\text { MERYL }} \stackrel{\text { top }}{\text { BICYCLE }}$ CL:X(palm down) BICYCLE-MOVE-FORWARD "Here's Meryl, and a bike went by."
}

In the case of (17) the example is marginal, in any event, and much improved by the addition of a CL-G ${ }^{9}$ or an index point, to locate Meryl, as in (18). However, once the person classifier is included, the generalization made above still holds.
18. $\frac{\text { top }}{\text { MERYL CL-G }}$
top
18. MERYL CL-G BICYCLE
"Here's Meryl, and a bike went by." 
Where there is both a person and an animal topic, the focus may be on the animal, and not necessarily on the person. This is shown in sentence (19) where there is an initial animate topic (MAN), followed by an animal NP in topic position. The following classifier construction is associated with the animal and not the person. In this case, DOG is the topic (and the subject) associated with the classifier predicate.

\section{9. $\frac{\text { top }}{\text { OTHER INDEX MAN }}$ top \\ "There's a man, and a dog, about to run."}

\section{Three place predicates}

In this section of the paper, we look specifically at those sentences in which the verb takes two internal arguments, as well as an external argument. In English, these are sentences such as : The man put the cup on the table or The man gave the woman a cup/ The man gave a cup to the woman. These sorts of sentences are of particular interest in signed languages, because in a certain sub-class of three place predicates, i.e., those involving movement or location, some aspect of the theme argument, i.e. cup, is necessarily incorporated into the predicate. In signed languages, it seems that the handshape of the predicate in sentences like (20) and (21) is the handshape that is used to classify the particular NP in theme position. Thus, the predicate in (20) will use the classifier handshape generally associated with cups $^{10}$, and the predicate in (21) will use the classifier handshape ${ }^{11}$ generally associated with book shaped objects as they are passed from one person to another.

A classifier predicate always consists of at least a movement and a handshape. In the case of classifier predicate constructions, the handshape always takes the form of the classifier used for the theme argument. The movement is between sources and goals (from $\mathrm{X}$ to $\mathrm{Y}$ ) or from one location to another. Thus, to show how this handshape incorporation works, we will use examples that differ only in terms of theme. 
top

20. MAN CL-G $\mathrm{G}_{\mathrm{i}} 12$

top

CL-Gj

WOMAN CL-Gj CUP

¡GIVE-CUP-SHAPED-OBJECT -

Man (over there) woman (over there) he gives cup her

"The man gives the woman a cup."

In this case the cup shaped object is represented by a $\mathrm{C}$ handshape. The $\mathrm{C}$ handshape moves from the location of MAN (indicated by ${ }_{i}$ ) to the location of WOMAN (indicated by ${ }_{j}$ ).

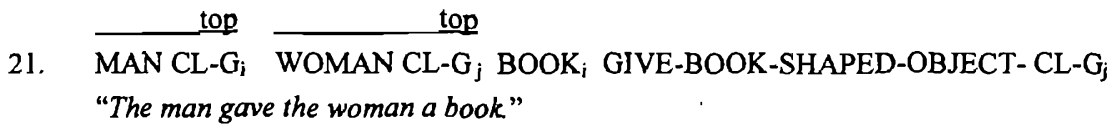

In this case the book shaped object is represented by a flat B handshape, palm up. The B handshape moves from the location of MAN (indicated by $i$ ) to the location of WOMAN (indicated by $j$ ).

\section{top top \\ 22. MAN G-CLi WOMAN G-CLj \\ COIN $i$ GIVE-COIN-SHAPED-OBJECT-CL-G $j$ \\ "The man gave the woman a coin."}

In this case the coin shaped object is represented by an $\mathrm{F}$ handshape in which thumb and forefinger make a small circle. The small circle handshape moves from the location of MAN (indicated by $i$ ) to the location of WOMAN (indicated by $j$ ).

Note that in these examples, the only difference is in the handshape that forms the complex classifier in conjunction with the movement from location $i$ to location $j$. In each case the handshape takes the form that represents the theme argument. Note also that the theme argument occurs as a full lexical NP. Strictly speaking then, we should say not that the theme argument has been incorporated, but that a morpheme representing the theme has been incorporated. In this sense, we may be talking about the handshape being an agreement morpheme, without which the movement is incomplete as a predicate. 
In any event, whether we refer to the handshape in these cases as a classifier, a class marker or an agreement marker, the fact is that the marker of the theme is necessarily incorporated into the movement frame in order to form a complex predicate. We look now to the syntactic positions that are available for each of the three arguments in a SASL sentence of the type we have been discussing. The examples that follow may all be translated as The man gave the woman a cup.

Note that we gloss elements that are non-manually marked with topic marking as top. As we will show, up to two of these elements in a sentence may be topic marked, but three may not. Items functioning pronominally, either as classifiers or classifier clitics indicating the location of different NPs in the signing. space are irrelevant to the level of analysis in this section, although we include them for completeness. The following sentences are grammatical.

top top

23. MAN CL-G $i$ WOMAN CL-G $j$ BOOK iGIVE-BOOK-CL $j$ top top

24. WOMAN CL-G $j$ MAN CL-G $i$ BOOK $i$ GIVE-BOOK-CL-G $j$

25. $\frac{\text { top }}{\text { MAN CL-Gi } i \text { top }}$ $i$ GIVE-BOOK-CL-G $j$ WOMAN $j$
top
top
MAN CL-G $i$
iGIVE-BOOKj CL-G $j$ WOMAN $j$

However, on the same reading, the following sentences are ungrammatical.

27. $\underset{\text { *BOOK }}{\text { WOMAN CL-G }}$ MAN CL-Gi $i$ GIVE-BOOK CL-Gj

28. $\frac{\text { top }}{\text { *WOMAN CL-G } j} \stackrel{\text { top }}{\text { BOOK }}$ MAN CL-Gi iGIVE-BOOK CL-G $j$

Thus it can be seen that in three place predicates such as GIVE-SOMETHING, if both topic positions are filled, they may not be occupied by both the direct and the indirect object. We 
might also say that both topic positions may not be filled by the theme and the goallocation arguments. In the case of the sentences above, all meaning The man gave the woman a book, the relative order of the topics, as long as they are not the direct and indirect object, in which case they are ruled out, is irrelevant, as far as grammaticality is concerned.

However, when we deal with sentences which have the English meaning The man put the cup on the table, there are indeed ordering restrictions. As shown above the direct and indirect object may not both occur in topic positions. Further, the figure may not precede the ground. Thus, TABLE (the location), must always be either in the first or second topic position.

Morphologically, the classifier predicate in the sentences meaning The man gave the woman a book may be said to have incorporated an agreement morpheme of the theme argument, as well as incorporating the locations of the source and goal arguments ${ }^{13}$. The source agreement is indicated spatially, whereas the goal agreement may be indicated both spatially and with the addition of a classifier clitic. In sentences meaning The man put the cup on the table, both the theme and the location arguments are encoded into the classifier predicate. Syntactically, thus, the classifier predicate forms a kind of minimal sentence unit. Its actual NPs are not specified in full, but the predicate has a syntax all of its own. It is possibly for this reason that one or two of the NPs are forced into topic positions, since to some extent they are syntactically redundant, when complex classifier predicates are present in an utterance.

The examples shown above to illustrate NP-verb agreement in sentences containing classifier predicates are not the only grammatical possibilities, although we have indicated the crucial ungrammatical cases. It is, in fact, extremely common for utterances, particularly in a naturalistic narrative context, to have two NP topics and for the signer to vary perspective from the action of a person to the movement of an object and back again, sometimes switching back and forth more than once or twice. This is a strategy that is entirely suited to the modality of a signed language. In a spoken language, one might embed a series of verbs into a sentence, as in (29), particularly to describe a continuous series of movements. However, there is a limit as to what an English sentence can do, without becoming awkward.

29. The man has jumped out of the plane and is falling feet first to the ground in a floating motion while the parachute opens up and balloons above him as he continues to float 
down held aloft by the ropes of the parachute which is swaying in the wind as he floats lightly towards the ground, his parachute collapsing on top of him.

Although the above example is possible in English, it is highly unlikely. In the first place, it is, in fact, a series of conjoined and embedded sentences. Secondly, English has ways of conveying the same information in a manner more suited to its modality. It is one of the advantages of the visual-spatial modality that it permits more than one piece of information to be conveyed at a time. It also enables the simultaneous presentation of more than one piece of information and the additional information that these things are actually happening simultaneously. Signed languages are also able to use perspective changes within one sentence (a phenomenon that does occur in spoken languages which use serial verb constructions, and in languages like English by using syntactic constructions introduced by while or at the same time.)

In our data, we found utterances such as the ones listed below involving two NP topics, in this case a person and a parachute, both nominal classifiers.

\section{NEXT (RH) CL-5 : PARACHUTE \\ (LH) CL-I: PERSON}

CL-S (2-H) PERSON-HOLD ROPES

CL-S (2-H) PERSON- HOLD-ROPES-LOOK-AROUND

Eyegaze sees plane
(RH) CL-Y PLANE FLY
(LH) CL-S HOLD ROPE

leans left to avoid being hit by plane

CL-S (2-H) PERSON HOLD ROPES

(RH) CL-Y PLANE FLY

(LH) CL-S HOLD ROPES

lean right to avoid being hit by plane

(2-H) CL-S HOLD-ROPES 
look down and continue leaning right

(2-H) CL-S HOLD ROPES

body moves from right to left

(RH) CL-V' PERSON FLOATS DOWN(bent legs)

(LH) CL-S HOLD PARACHUTE ROPE

Essentially, through most of this utterance, there are two classifier predicates. The one is the classifier predicate meaning "a plane is flying", and the other is the classifier predicate meaning "the man is holding on to a parachute's ropes and descending to earth." Right before the end there is a third classifier predicate introduced, and that is the one meaning "a person floats down to earth." There is a good deal more information conveyed than in the meanings we have presented.

There are several issues that must be raised when we find data like these. Firstly, such data are frequently found in naturalistic discourse. Secondly, we need to ask, "Is this a single sentence?". Thirdly, if it is a single sentence, how do we analyse it? And finally, to what extent are we dealing with a grammatical system and to what extent are we talking about role-play? If we are talking about role-play, what are the grammatical constraints on the occurrence of this roleplay?

One might wonder what the purpose of asking the first question is. Does it matter if it is one sentence or many? We think it does matter, because it is important to establish the minimal syntactic possibilities, although never possible to establish the upper limit. We have shown above what the minimal possibilities are. Now it is important to find out what else is possible in a single sentence. So, in answer to the first question, "Is this a single sentence?" we think we must answer yes, although it is undoubtedly a sentence with a good deal of verb embedding. It has a single topic, the man who is parachuting. The man, while parachuting, sees a plane fly past. The man tries to avoid being hit by the plane and floats down to earth.

Grammatically, the generalization we proposed above, holds in this utterance. The agreement with person holds throughout the utterance. The signer uses a two handed $\mathrm{S}$ handshape to show the person holding the ropes. This is a well-formed sign, involving the handshapes used for a 
person holding something, and it remains throughout the utterance. The signer dramatizes whilst holding the ropes, for instance, he looks around, he sees a plane, he leans to avoid being hit by a plane. In the meantime, while maintaining the sign for holding the ropes, he signs (using a classifier) that a plane flies by, as seen from the perspective of the parachutist, and finally he uses another classifier person handshape to show a person floating down. Note that the handshape HOLD-ROPES continues throughout the utterance, once it is introduced. When the signer needs to show other things that are happening at the same time, he uses his non-dominant hand to maintain the sign HOLD-ROPE.

We think it is reasonable to claim the CL-S HOLD-ROPE is part of the grammatical system, and is the main verb in this utterance. It is also clear that CL-Y PLANE-FLY is part of the grammatical system too, and is a complex verb form that co-occurs with the CL-S HOLD ROPE. The same holds for CL-bent V PERSON-FLOATS DOWN. Depending on one's grammatical theory, one could analyse the verbal constituents of the sentence in different ways, but it is possible in any theoretical framework to represent these verbs and their relationship to one another. Signed languages apparently do not put an upper limit on the number of iterations of these alternating verb forms. The single requirement is that the person involvement, in terms of classifier handshape agreement must occur either concurrently with other verbal information, flanking it or sandwiched between it.

\section{Role Play}

The next question is about the status of role-play, the involvement of the signer's body as part of taking up the role of the parachutist. We have deliberately avoided glossing places where we think this happens as role-play, as this prejudges the question. In this case, we think it is obvious that the signer assumes the role of the parachutist. This is still within the grammatical system -- it means he signs from first person perspective. Note however, that although the signer uses his eyes (looking at the plane) and his head (leaning to avoid being hit by the plane) mimetically, he does this concurrently with the sign HOLD-ROPE. This additional dramatization is happening concurrently with a linguistic item, i.e., the classifier predicate HOLD-ROPE. Finally in the last part of the utterance, the signer signs CL-V PERSON FLOAT DOWN concurrently with HOLDROPE, a sign language-specific way of merging both perspectives. This construction is sometimes called SAME-TIME-WHILE in the literature, and has been discussed, among others by Liddell, 1984. 
At this point then, using the examples we have, we can say that if there is role-play it co-occurs with classifier predicate forms. We need however to look at some other examples to see if this is always the case, or just one part of the story.

In utterance (31) below, the signer is describing a woman pushing a baby in a baby carriage (pram). Apparently, the signer first sets up two of the lexical NPs as topics, then uses a classifier predicate construction to show the relationship of the agent, the patient and the location. Thereafter, the signer assumes the role of the baby. The signer then produces an extended roleplay in which, as baby, he looks up, waves his arms about, and mimes gurgling. He then switches role back to the woman, and then uses a classifier construction for pushing a carriage.

\section{rha top top \\ 31. NEXT WHAT WOMAN BABY CL 5: PERSON-PUT-BABY-IN-PRAM BABY-WAVE-ARMS-GURGLE CL:S PERSON-PUSH-PRAM}

In this case, WOMAN is the main person topic (also the agent), and BABY is the second topic (and happens to be the theme). The signer first assumes the role of the woman putting the baby in the carriage, using a classifier predicate, then switches role to the perspective of the baby, using 2-H S fists, and a baby's facial expression, then switches again to the woman, pushing the carriage, using the 2-H CL-S handshape. The crucial question, we believe, is not whether the actions of putting the baby into the carriage or pushing the carriage are classifier predicates, but what the status of the role-shift into BABY is. One might want to say that the 2-h S-handshapes are classifiers for the baby's fists, but it seems more likely that this is role-play, flanked on either side by classifier predicates.

A similar example can be seen in (32). The topic is PEOPLE, (in a crowd). The crowd moving forward is shown by a classifier predicate using a spread 5 handshape. The signer then shows the crowd walking and talking. Then he adopts the role of one person in the crowd and uses his whole body to mime walking, waving and bumping against others. Thereafter, he uses the classifier predicate CROWD-WALK FORWARD TALK to flank the mime or role play. The reason we claim that this is role play is firstly that the signer uses his whole body, and secondly that he changes person to focus on one rather than many, as well as changing perspective. Thus 
the role play does not have a grammatical antecedent in the way that classifier predicates always do, in terms at least of number.

\section{NEXT PEOPLE CROWD CL:5 CROWD-WALK-FORWARD+ WALK TALK WAVE BUMP CL:5 CROWD-WALK-FORWARD}

This is not the only way of signing an utterance with the meaning "the people were walking along and talking". Another informant produced (33), in which the only role-play involves some miming of the movement of the head to talk to the person next to him accompanying the sign TALK.

\section{top top \\ 33. FATHER MOTHER 2-H CL:X PERSON-WALK ME TALK 2-H CL:X PERSON-WALK-FAR+++}

When pressed for his intuitions, one of our informants said that for him, (34) was the best way of conveying the same meaning, but that (32) and (33) were fine. Sentence (34) does not have what we have been calling role-play, however, note that this is much the same as the example in (30), with the two verbs co-occurring with, as well as being flanked by, the classifier predicate (abstracting away from some of the details).

top
34. PEOPLE 2-H CL:5 CROWD-WALK
(RH) CL:5 CROWD-WALK
(LH) TALK

\section{2-H CL:5 CROWD-WALK}

In (35) we see an example, of role-playing in sentence-final position, following a classifier.

35. INDEX ONE MAN ALONE WHAT. MMM OLD 2-H CL-5 BEER BELLY (X2) MAN WALKS -SLUGGISHLY

In (35), the signer uses his whole body to mime walking sluggishly. Note however, that this role play follows the size and shape classifier that follows the outline of his belly. 
In (36) we see several iterations in which the perspective changes, focusing on parts of the dog, such as his ears, his tail, his leash and his collar. These are all classifier constructions. However, note that when the signer is signing EARS, he assumes the role of the dog, as we can see by his facial expression. Thus, in this case, role-play occurs concurrently with the classifier construction.

\section{NEXT WHAT DOG (2-H) CL-B EAR RH CL-F LEASH facial expr.dog LH CL-C COLLAR}

(2-H) CL-B EAR

facial expr. dog

\section{RH CL-1 TAILWAG+H \\ LH CL-B DOG'S BODY}

Sentence (36), which is of a type commonly appearing in signed languages, presents problems for any sort of traditional sentence analysis that has been proposed for spoken languages. However, it clearly obeys the rudimentary grammatical constraints that we have outlined in this paper, and may thus be said to be rule-governed. Role-play elements may only occur within the constraints of the grammar, and not haphazardly. Certainly, however, there is much work to be done before we can say that we have adequately analysed sentences like this.

Thus, we claim that when role-play, mime and gesture do occur in sentences involving classifier constructions, they obey very specific grammatical constraints 14 . They may only follow NPs in topic position, and they can never occur before the initial signing of the classifier predicate. They must either follow a classifier predicate, or they must occur simultaneously with a classifier predicate. Role-plays that follow a classifier predicate may also themselves be followed by a repetition of the initial classifier predicate. 


\section{Conclusion}

In this paper we have looked at two aspects of constructions involving classifier handshapes in interaction with the syntax of an utterance. The first aspect we examined is the requirement that in signed languages, classifier predicates are preceded by at least one element in the topic position of a sentence. We explored the syntactic constraints on sign order in the use of classifier predicates in SASL. Further, we examined the syntactic relationship between classifier predicates and the items occurring in topic positions.

The second aspect we examined is the interaction of classifier predicates and role-play. We differentiated the use of the signer's hands to make handling (Supalla 1982) or instrumental (Liddell and Johnson 1987) and body part (Supalla 1982) classifier constructions as part of the complex predicate from role play. These can be distinguished from role-play, because the occurrence of the handshape may be seen as adding semantic meaning and morphological agreement to the complex verb frame.

We attempted to define what phenomena do constitute role-play in signed languages. Thereafter, we described and attempted to explain the constraints on the occurrence of role-play within utterances containing classifier predicates. We argue that role-play is not actually a part of the grammar, although we show that its occurrence, particularly in utterances involving classifier predicates is subject to grammatical constraints. We show that when role-plays occur within utterances involving classifier predicates, they can never occur before a classifier predicate. They can only occur in the following positions:

(a) flanked by classifier predicates,

(b) simultaneously produced with a classifier predicate,

(c) following a classifier predicate

Clearly, the interaction of classifiers and syntax is still a most unexplored area, and what we have sketched here is only a very preliminary description. Data are required from other signed languages, and we need to incorporate research on the interaction of gesture and syntax in general. 
NOTES

1 We are grateful to our consultants, Helen Morgans, David Pedro, Gavin Johnson, Troy Panaino, and Natasha Mohammed, as well as to Karen Emmorey and the participants at the Classifier Workshop in San Diego CA in April 2000 for helpful comments and questions. We particularly thank Adam Schembri for extremely helpful comments on a version of this paper. Where we have not followed advice, of course, we are entirely responsible.

The funding for this research was provided by grant number 15/1/3/16/012 from the Centre for Science Development of the Human Research Council of South Africa, a research grant from the University of Stellenbosch, and travel funding from the National Science Foundation. The Deaf Federation of South Africa (DEAFSA) provided the camera and computer equipment for the Gauteng research site.

2 We use the term, "classifier predicate" as proposed by Liddell (1977). We consider the term to refer to the same class of verbs that Supalla (1982) calls "verbs of motion and location". We use the terms"complex verb", "complex verb form", "complex verb frame"", interchangeably with the term "classifier predicate" in this paper. We are aware of the debate surrounding this terminology, and in all cases, we are referring to what in the literature of signed languages for some time have been referred to as classifier predicates. We take no position on whether this term is accurate or not, we simply use it alongside the others, in the hope that readers will identify the phenomenon or set of phenomena to which we are referring.

3

We refer to the position on the left-most edge of the sentence, external to the sentence itself, containing left-dislocated or topicalised elements, as the topic position. Thus, in an utterance glossed as:

\section{VEGETABLE, JOHN LIKE SPINACH}

"As for vegetables, John likes spinach".

we refer to the position in which VEGETABLE occurs as the topic position.

4 This is generally accepted by sign language linguists. See Aarons 1994 for a fuller discussion.

5 The videotaped data are part of a large project to investigate variation in the signed language used in South Africa. The data have been elicited from a total of seventy signers in seven distinct Deaf communities located in two geographical areas of South Africa. An elicitation task designed to elicit classifier constructions and certain syntactic structures was used. The elicitation task consists of 22 cartoons, and signers are asked by a fluent Deaf signer of their own community to sign (on camera) what they see in the cartoons. 
6 In fact, the topic position nearest to the clause, seems to us to be occupied by focus elements as a general rule. This claim is beyond the scope of this paper, but it is entirely plausible, in light of a proposal by Rizzi 1997, that focused elements occur nearest to the clause, and can themselves be preceded by topic elements.

7

All the examples are from SASL. Since the internal morphological structure is irrelevant to the syntactic claims being made here, we are presenting these glosses only at the level necessary for our analysis/description and are abstracting away from many of the important morphological details here.

8 It is also possible to find utterances such as $\frac{t}{\text { MERYL }} \quad \frac{t}{\text { BICYCLE }}$ CL-BENT V on CL-X (person on bicycle) MOVE FORWARD In this case, the entire verb complex is made up of two classifiers, and the person classifier moves forward as part of the whole unit.

${ }^{9}$ Although we have not specifically discussed the appearance of classifiers in Topic Position, we concur with Wallin (this volume) that these are nominal classifiers.

10

a $\mathrm{C}$ handshape, palm orientation perpendicular to the ground.

11

a B handshape, palm up.

12 The classifiers used in apposition to the full NPs here are those referred to in the note above. These are discussed at length by Wallin (in press).

13 Liddell (in press) has presented arguments against considering location or direction as morphological. He does not dispute the morphological status of the handshape itself. We believe that some incorporation of the representation of goal and source occurs in these sorts of predicates. If there are compelling reasons not to call these representations morphemes, we will defer to those reasons.

14 We are not saying that role-play, mime, and gesture do not occur in contexts where they do not co-occur with classifier predicates, nor are flanked by them, we are only discussing the cases where they do. 


\section{REFERENCES}

Aarons, D.1994. Aspects of the Syntax of American Sign Language. Unpublished doctoral dissertation. Boston University. Boston, MA.

Aarons, D. 1996. Topics and Topicalization in American Sign Language. In SPIL 26, Stellenbosch Papers in Linguistics 26, 65-106. University of Stellenbosch: South Africa

Aarons, D., R. Morgan, and H. Morgans. 1998. South African Sign Language after Apartheid. Sixth International Conference on Theoretical Issues in Sign Language Research, Washington, DC.

Engberg-Pedersen, E. 1993. Space in Danish Sign Language: the semantics and morphosyntax of the use of space in a visual langriage. Hamburg: Signum Press.

Shepard-Kegl, J. A. 1985. Locative relations in American Sign Language word formation, syntax and discourse. Unpublished doctoral dissertation, Massachusetts Institute of Technology. Boston, MA.

Liddell, S.K. 1984. Unrealized inceptive-aspect in American Sign Language: Feature insertion in syllabic frames. In Drago, J., V. Mishra, and D. Teston (eds.). Papers from the $20^{\text {th }}$ regional meeting of the Chicago Linguistic Society. Chicago: University of Chicago Press.

Liddell, S.K., and Robert E. Johnson. 1987. An analysis of locative predicates in American Sign Language. Fourth International Symposium on Sign Language Research. Lapeenranta, Finland.

Liddell, S.K. and M. Metzger. 1998. Gesture in sign language discourse. Journal of Pragmatics 30: 657-697.

Rizzi, L. 1997. The fine structure of the left periphery. In L. Haegeman (ed.). Elements of Grammar. Dordrecht: Kluwer Academic Publishers.

Schembri, A. (in press). Rethinking classifiers in signed languages. In Emmorey, K. (ed). Perspectives on classifier constructions in sign languages. NJ: Erlbaum.

Supalla, T. 1982. Structure and acquisition of verbs of motion and location in American Sign Language. Unpublished doctoral dissertation. University of California, San Diego. San Diego, CA.

Supalla, T. 1990. Serial verbs of motion in ASL. In S. Fischer and P. Siple (eds.). Theoretical Issues in Sign Language Research: Linguistics . Chicago: Chicago University Press.

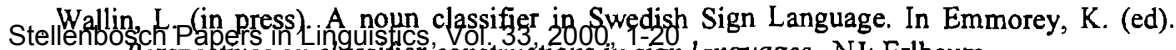

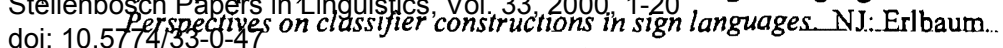

